\title{
Weibull Deterioration Rate on Integrated Cold Chain Inventory Performance
}

\author{
Jin-Jin Mou, ${ }^{1, *}$, Ying-Mei Jiang ${ }^{1}$ \\ ${ }^{1}$ School of Management, Shandong Vocational University of Foreign Affairs, Weihai 264504, China
}

\begin{abstract}
Different from the previous articles on multi-echelon deteriorating inventory, this paper explains the economic implications of Weibull parameters for cold chain inventory rather than restricting to mathematical sense. A case study shows that DC warehousing activities and total throughput of the scale factor has a significant effect on the total profit compared with the storage technology and warehousing environment of shape factor. The improvements of DC warehousing standard and total throughput and preservation technology and warehousing environment contribute to the reduction of some DC operation costs and the increase of DC processing and customizing profit.
\end{abstract}

\section{Introduction}

On the deteriorating rate of cold chain product, most of the current works thought of as a constant rate or instantaneous deterioration (Yang, Wee 2000; Das et al., 2015; Duan et al., 2018; Mahmoodi et al., 2019). However, its shelf life, quality and safety throughout the supply chain is greatly impacted by warehousing environmental factors, especially temperature and relative humidity (Aung \& Chang 2014; Yang et al., 2020) and warehousing technology (Huang et al., 2018). Thus, some cold chain products, such as, food, fruits, and vegetables are not decayed instantaneously. Weibull non-instantaneous deterioration function is used to depict the deteriorating characteristics of cold chain products (Yang 2012; Dye 2013; Sanni, Chukwu 2016).

In the comparison of the performance of three-echelon cold chain system with two echelon system, threeparameter The distribution center as a third-party logistics that efficiently links the logistics of producers and retailers and consolidates all parties into a system, can not only enhance the core competencies of DC warehousing, storage and distribution, but also improve the system profitability as a result of perfectly applying with customers' requirements for small quantity with large variety and joint operation for all customers, with its value added services of product processing and customizing, picking and assorting.

Motivated by the above background, following questions would be proposed: How do the DC deterioration parameters affect the integrated cold chain inventory performance? And how do the DC deterioration parameters affect the DC cost structure and its gross profit.

The rest of this paper organized as follows. Section 2 presents the problem description. Section 3 provides numerical analysis. Finally, it summarizes the findings and concludes the potential impacts of this work in section.

\section{Problem description}

In this paper, three-echelon integrated cold chain inventory system composed of three manufacturers (expressed as), one DC and two retailers (expressed as), with non-instantaneous Weibull deterioration rate items in a finite horizon is investigated and proposed to obtain the optimal order quantity with an objective of maximizing the system profit.

Different from two parameter Weibull distribution, three parameter Weibull function can more comprehensively fit the deterioration rate of different conditions. The density function and cumulative distribution function of three parameter Weibull distribution for cold chain products can be written as $f(t)=\alpha \beta(t-\gamma)^{\beta-1} e^{-\alpha(t-\gamma)^{\beta}}$

$F(t)=1-e^{-\alpha(t-\gamma)^{\beta}} \quad$,respectively, and the noninstantaneous Weibull deterioration rate is $\theta(t)=\frac{f(t)}{1-F(t)}=\alpha \beta(t-\gamma)^{\beta-1}$, which is the deterioration trend of the cold chain product at time $t$. Of which, $\alpha$ is the scale parameter $(\alpha>0), \beta$ is the shape parameter $(\beta>0)$ and $\gamma$ is the position parameter $(\gamma>0)$ respectively. The curve shape is shown in Fig. 1

\footnotetext{
*Corresponding author: mujinjinde@163.com
} 


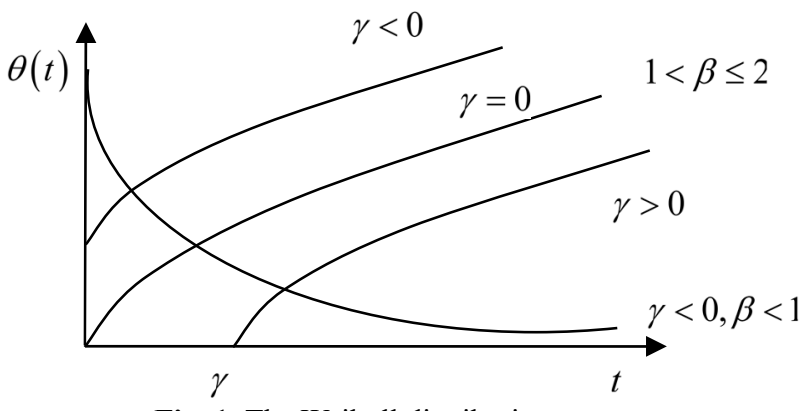

Fig. 1. The Weibull distribution curves.

Through the deterioration rate function of $\theta(t)$, we can know that, if $\alpha=0$, then $\theta(t)=0$, that is, there is no deterioration of goods; if $\alpha>0$, there is deterioration of goods. If $\beta=0$, the deterioration rate is a fixed constant. As per Fig. 1 , if $1<\beta \leq 2$, the curve shows an upward trend, if $0<\beta<1$, the curve shows a downward trend. While the position factor $\gamma$ determines the starting position of product deterioration. If $\gamma=0$, it means that deterioration occurs immediately once the cold chain products enters the distribution center (DC). If $\gamma>0$, it means that there is still a period of freshness-keeping period after the goods enter the DC, that is, there is a noninstantaneous deterioration. Similarly, if $\gamma<0$, it means that the product has occurred deterioration for a period of time before its entering the DC. Due to the different combinations of the three parameters in Weibull distribution, the deterioration rate presents different function curves. Compared with fixed or linear deterioration rate, Weibull function can better describe the deterioration characteristics of different cold chain products. In this paper, the parameters discussed are as $\alpha>0,1<\beta \leq 2, \gamma>0$. Especially, we will analyse the effect of $\alpha_{d k}$ and $\beta_{d k}$ on DC cost and system profit.

\section{Case study and analysis}

This paper is based on the study of three-echelon cold chain inventory system (Wang et al., 2017) to examine the effect of Weibull distribution factors of DC on system profit, so, inventory model formulation and numerical calculation are ignored. Through investigating a local cold chain system, the regarding parameters and system profit are listed in Table 1-2. We only list the major parameters with Weibull distribution factors and DC related factors. The meanings of the DC related parameters are: (1) $h_{d k}$ : holding cost per unit time of DC for theth product; (2) $G_{d}$ : picking and assorting cost per order of DC; (3) $v_{d k}$ : delivery cost ratio of DC for theth product and (4) $E_{d k}$ : unit processing and customizing cost per for the for the kth product .

Table 1. Parameter data of the DC.

\begin{tabular}{|c|c|c|c|c|c|c|c|}
\hline & $\alpha_{d k}$ & $\beta_{d k}$ & $\gamma_{k}$ & $G_{d}$ & $E_{d k}$ & $h_{d k}$ & $v_{d k}$ \\
\hline$k=1$ & 0.01 & 1.5 & 0.5 & \multirow{3}{*}{1500} & 1 & 3 & $8 \%$ \\
\cline { 1 - 5 } \cline { 6 - 8 } & 0.01 & 1.4 & 0.5 & 0.8 & 2 & $7 \%$ \\
\hline$k=3$ & 0.01 & 1.3 & 1 & & 0.5 & 0.5 & $8 \%$ \\
\hline
\end{tabular}

Table 2. The profit of supply chain.

\begin{tabular}{|c|c|c|c|c|c|}
\hline & $k=1$ & $k=2$ & \multicolumn{2}{|c|}{$k=3$} & \multirow{2}{*}{ Summation } \\
\hline & $i=1$ & $i=2$ & $i=1$ & $i=2$ & 10.3471 \\
\hline Retailer & 3.8206 & 4.5546 & 0.9664 & 1.0055 & 3.3246 \\
\hline DC & \multicolumn{3}{|c|}{3.3246} & 7.5333 \\
\hline Producer & 3.5406 & 3.5152 & \multicolumn{2}{|c|}{0.4775} & 21.205 \\
\hline Summation & $/$ & $/$ & $/$ & \\
\hline
\end{tabular}

Note: The real data $=$ the data in the table $\times \mathrm{e}+004$.

In a cold chain, as is defined, the effect of DC warehousing activities and its total throughput on deterioration is called a scale factor of Weibull distribution and DC storage technology and warehousing environment on the effect of deterioration is called a shape factor of Weibull distribution. Here, the key parameters of the DC deterioration rate $\alpha_{d k}$ and $\beta_{d k}$ are chosen for sensitivity analysis.

The optimal profits are obtained in Table 3 and Table 4 by varying the parameters' values of $\alpha_{d k}$ and $\beta_{d k}$ from $-40 \%$ to $40 \%$, respectively. 
Table 3. The effect of $\alpha_{d k}$ on system profit.

\begin{tabular}{|c|c|c|c|c|}
\hline$\alpha_{d k}$ & $\pi_{b}$ & $\pi_{d}$ & $\pi_{p}$ & $\pi$ \\
\hline$+40 \%$ & 103095 & 35738 & 69573 & 208406 \\
\hline$+20 \%$ & 103095 & 37018 & 70060 & 210173 \\
\hline 0 & 103471 & 33246 & 75333 & 212050 \\
\hline$-20 \%$ & 103810 & 36658 & 73894 & 214362 \\
\hline$-40 \%$ & 103810 & 37828 & 74327 & 215965 \\
\hline
\end{tabular}

Through Table 3 and Table 4, it can be found that the total profit goes inversely with the change of DC deterioration rate. The increase of the deterioration rate caused by the warehousing activities and total throughput may be from frequent and improper handling and large throughput. The increase of the storage technology and warehousing environment factor may be from an improper or outdated DC preservation technology. All of which will lead to loss and damage of the deteriorating items, in turn, a decrease of total system profit. On the contrary, the improvements of DC warehousing standard and total throughput, and its preservation technology increase the system profit and certainly help realize the industry

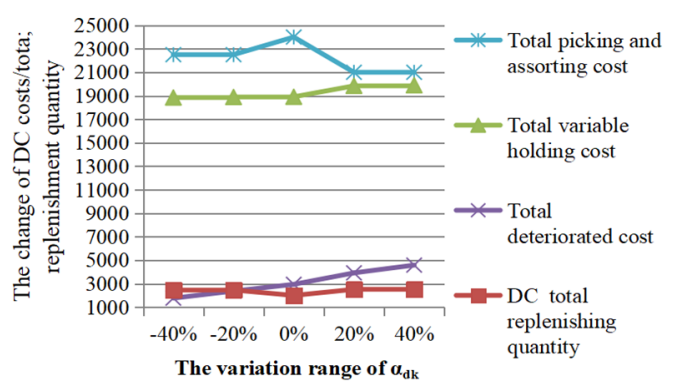

Fig. 2. The effects of $\alpha_{d k}$ on DC costs.

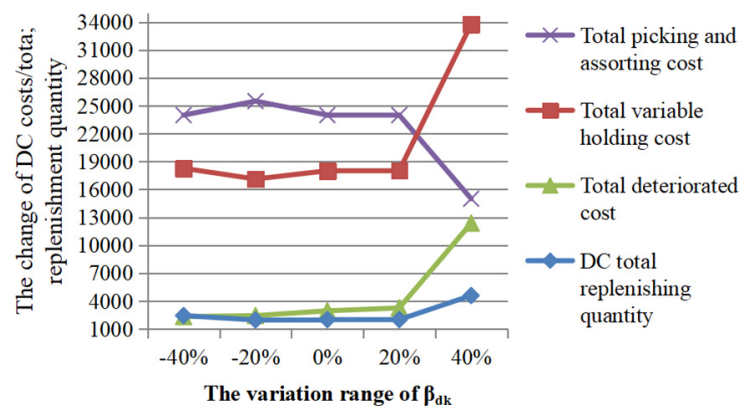

Fig. 4. The effects of $\beta_{d k}$ on DC costs.

Fig. 2 and Fig.4 demonstrate that the decrease of $\alpha_{d k}$ and $\beta_{d k}$ results in the decrease of the total deteriorated cost, the total variable holding cost, the decrease of total replenishing quantity and the increase of DC picking and assorting cost. It is worth noting that the increase of DC
Table 4. The effect of $\beta_{d k}$ on system profit.

\begin{tabular}{|c|c|c|c|c|}
\hline$\beta_{d k}$ & $\pi_{b}$ & $\pi_{d}$ & $\pi_{p}$ & $\pi$ \\
\hline$+40 \%$ & 99967 & 40458 & 68369 & 208794 \\
\hline$+20 \%$ & 103472 & 32645 & 75168 & 211285 \\
\hline 0 & 103471 & 33246 & 75333 & 212050 \\
\hline$-20 \%$ & 104186 & 30888 & 78366 & 213440 \\
\hline$-40 \%$ & 104206 & 33872 & 75704 & 213782 \\
\hline
\end{tabular}

structure optimization.

By comparing the effects of $\alpha_{d k}$ and $\beta_{d k}$ on the total profit, it can be found that DC warehousing activities and total throughput factor has a higher impact on the total profit than that of storage technology and warehousing environment factor. Thus, DC warehousing standard and process as well as the design of total throughput should be made and executed to guarantee the quality of the perishable products as a result that losses and damages are reduced simultaneously.

The effects of $\alpha_{d k}$ and $\beta_{d k}$ on DC cost and its gross profit are illustrated in Fig.2-5.

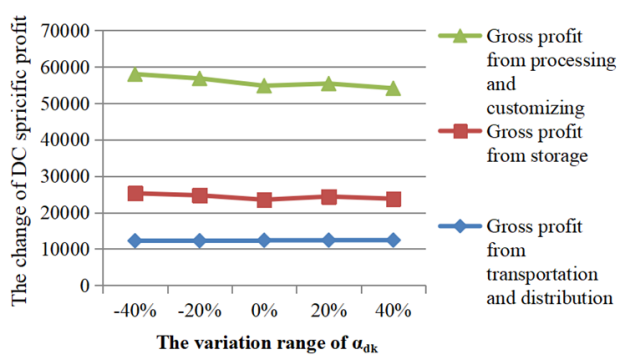

\#

Fig. 3. The effects of $\alpha_{d k}$ on DC service gross profit.

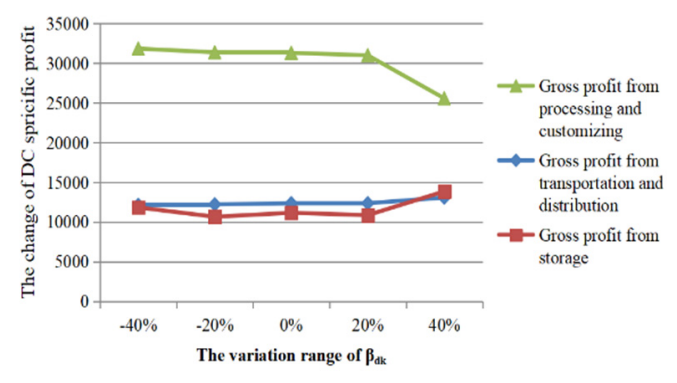

Fig. 5. The effects of $\beta_{d k}$ on DC service gross profit.

picking and assorting cost may be from the careful handling of the perishable products to assure the decrease of $\alpha_{d k}$ and may be from keeping an orderly and clean environment to assure the decrease of $\beta_{d k}$.

Fig. 3 and Fig. 5 show that DC gets more gross profit from the product processing and customizing service for 
the increase of available products owing to the decrease of $\alpha_{d k}$ and $\beta_{d k}$.

It is worth noting that, when $\beta_{d k}$ is increased by $40 \%$, DC total profit is the highest. The possible reason is that DC does not invest advanced preservation technology in the warehousing system, the cost could be reduced, in turn its profit is increased in a short term. But from the long run, distribution center should invest in preservation technology to devote to the improvements of the warehousing condition and the realization of industry structure optimization.

Thus, from the long run, both the improvements of DC warehousing standard and total throughput, and innovations in its preservation technology and environment could also highlight DC performance of the value-added services.

\section{Conclusion}

This paper proposes an integrated three-echelon cold chain inventory model with three-parameter Weibull deteriorating items. The major contributions are: (1)the economic implications of Weibull deterioration parameters are described to better display the effects of warehousing activities and total throughput, preservation technology on the deterioration rate and on the total system profit. That is, with the decrease of DC deterioration rate, the system profit is increased, of which, $\alpha_{d k}$ has a greater effect than that of $\beta_{d k}$. (2) The improvements of DC warehousing standard and total throughput and preservation technology and warehousing environment contribute to the reduction of some DC operation costs and the increase of DC processing and customizing profit.

These conclusions present good theoretical and managerial insights that DC warehousing activities should be carefully operated without rough handling, and the total throughput should be adaptable rather than overcapacity, DC processing and customizing service for all kinds of products and all logistics services including both the traditional service and value added services for high value products should be strengthened, meanwhile cost savings must be achieved from picking/assorting service to devote to higher profits for both total system and all members. More attention should also be paid to preservative technology innovation in DC and DC processing and customizing cost control.

This study could be extended to inventory-joint distribution policy and coordination inventory policy.

\section{Acknowledgement}

This research was supported by the National Natural Science Foundation of China Coordination and optimization of multi-echelon cold chain inventory system under compensation mechanism (71672166) and Shandong Social Science Planning Project Research on the governance structure and optimization of fresh overseas warehouse of cross border E-commerce (21CPYJ27)

\section{References}

1. Yang, P.C., Wee, H.M., 2000. Economic ordering policy of deteriorated item for vendor and buyer: an integrated approach. Production Planning \& Control, 11 (5): 474-480.

2. Das, B.C., Das, B., Mondal, S.K., 2015. An integrated production inventory model under interactive fuzz credit period for deteriorating item with several markets. Applied Soft Computing, 28 (C): 453-465.

3. Duan,Y., Cao, Y., Huo, J., 2018. Optimal pricing, production, and inventory for deteriorating items under demand uncertainty: The finite horizon case. Applied Mathematical Modelling,58:331-348

4. Mahmoodi A., 2019.Joint pricing and inventory control of duopoly retailers with deteriorating items and linear demand. Computers \& Industrial Engineering, 132:36-46

5. Aung, M.M., Chang, Y.S., 2014. Temperature management for the quality assurance of a perishable food supply chain. Food Control, 40 (1):198-207.

6. Yang, Y., Chi, H., Zhou, W., Fan, T., Piramuthu, S., 2020. Supply chain inventory-transportation optimal model under discount for perishable items. Decision Support Systems, 134: 113308

7. Huang, H., He, Y., Li, D., 2018. Pricing and inventory decisions in the food supply chain with production disruption and controllable deterioration. Journal of Cleaner Production 180:280-296

8. Yang, H. L., 2012. Two-warehouse partial backlogging inventory models with three-parameter Weibull distribution deterioration under inflation. International Journal of Production Economics, 138 (1): 107-116.

9. Das, B.C., Das, B., Mondal, S.K., 2013. Integrated supply chain model for a deteriorating item with procurement cost dependent credit period. Computers \& Industrial Engineering, 64 (3): 788-796.

10. Sanni, S.S., Chukwu, W.I., 2016. An Inventory Model with Three-Parameter Weibull Deterioration, Quadratic Demand Rate and Shortages. American Journal of Mathematical \& Management Sciences, 35 (2): 159-170.

11. Wang, S., Jiang, Y., Wang, X.,2017. An inventory model for the integrated three-echelon cold chain with a distribution center. Journal of systems \& management, 26(3):390-398. 